\title{
A nationwide survey on the perceptions of general surgeons on artificial intelligence
}

\author{
Frank J. Voskens ${ }^{1,4}$, Julian R. Abbing ${ }^{1,4}$, Anthony T. Ruys ${ }^{2}$, Jelle P. Ruurda ${ }^{3}$, Ivo A. M. J. Broeders ${ }^{1,4}$ \\ 'Department of Surgery, Meander Medical Center, Amersfoort 3813TZ, The Netherlands. \\ ${ }^{2}$ Department of Surgery, Elisabeth TweeSteden Ziekenhuis, Tilburg 5042AD, The Netherlands. \\ ${ }^{3}$ Department of Gastro-intestinal and oncologic Surgery, University Medical Center, Utrecht 3584CX, The Netherlands. \\ ${ }^{4}$ University of Twente, Robotics and Mechatronics, Enschede 7522NB, The Netherlands.
}

Correspondence to: Frank J. Voskens, Department of Surgery, Meander Medical Center, Maatweg 3, Amersfoort 3813TZ, The Netherlands. E-mail: frankvoskens@gmail.com

How to cite this article: Voskens FJ, Abbing JR, Ruys AT, Ruurda JP, Broeders IAMJ. A nationwide survey on the perceptions of general surgeons on artificial intelligence. Art Int Surg 2022;2:8-17. https://dx.doi.org/10.20517/ais.2021.10

Received: 8 Nov 2021 First Decision: 3 Dec 2021 Revised: 6 Dec 2021 Accepted: 20 Dec 2021 Published: 4 Jan 2022

Academic Editors: Andrew A. Gumbs, Roland Croner Copy Editor: Yue-Yue Zhang Production Editor: Yue-Yue Zhang

\begin{abstract}
Aim: Artificial intelligence (AI) has the potential to improve perioperative diagnosis and decision making. Despite promising study results, the majority of Al platforms in surgery currently remain in the research setting. Understanding the current knowledge and general attitude of surgeons toward Al applications in their surgical practice is essential and can contribute to the future development and uptake of $\mathrm{Al}$ in surgery.
\end{abstract}

Methods: In March 2021, a web-based survey was conducted among members of the Dutch Association of Surgery. The survey measured opinions on the existing knowledge, expectations, and concerns on Al among surgical residents and surgeons.

Results: A total of 313 respondents completed the survey. Overall, $85 \%$ of the respondents agreed that Al could be of value in the surgical field and $61 \%$ expected Al to improve their diagnostic ability. The outpatient clinic (35.8\%) and operating room (39.6\%) were stated as area of interest for the use of Al. Statistically, surgeons working in an academic hospital were more likely to be aware of the possibilities of $\mathrm{Al}(P=0.01)$. The surgeons in this survey were not worried about job replacement, however they raised the greatest concerns on accountability issues $(50.5 \%)$, loss of autonomy (46.6\%), and risk of bias (43.5\%). 
Conclusion: This survey demonstrates that the majority of the surgeons show a positive and open attitude towards Al. Although various ethical issues and concerns arise, the expectations regarding the implementation of future surgical Al applications are high.

Keywords: Artificial intelligence, surgery, survey

\section{INTRODUCTION}

Since the early 1950s Artificial Intelligence (AI) has been broadly defined as the ability for machines to reason and perform cognitive functions that we associate with human thinking such as decision-making, problem-solving, and learning ${ }^{[1,2]}$. In the past decade, novel artificial intelligence technologies have made remarkable progress with the further evolution of machine learning and deep learning algorithms ${ }^{[3]}$. This offered the opportunity for autonomous learning, of new pattern recognition and relationships. Deep learning has become a component in many areas of everyday life, with applications ranging from virtual assistants and speech recognition in mobile phones to self-driving $\operatorname{cars}^{[4]}$.

Digitalization in medicine has stimulated the research and development of artificial intelligence applications in healthcare. In particular, the widespread adoption of electronic health records, digital imaging, and digital diagnostic tests has provided large datasets suitable for computerized algorithms ${ }^{[5]}$. AI research has already been conducted in numerous medical fields and can revolutionize medicine by improving treatments, outcome prediction, and efficiency ${ }^{[6]}$. AI-powered diagnostic algorithms have shown high-level performance in the field of radiology ${ }^{[7-9]}$, pathology ${ }^{[10,11]}$, ophthalmology $y^{[12,13]}$, and dermatology $y^{[14,15]}$, but to date the utilization in the surgical field remains limited. The etiology is likely multifactorial, including the surgical environment with complex human interaction, lack of perceived necessity, and the limited knowledge and evidence of AI applications in the surgical practice ${ }^{[16]}$. With ongoing advances in computational power and robotic platforms, surgeons have the opportunity to play a critical role in the further rise and dissemination of AI in hospitals. Several surgical AI platforms have been reported in trials, with advancements in preoperative diagnosis and decision making, outcome prediction, surgical planning, and intraoperative surgical guidance ${ }^{[17]}$. Despite the promising results, these AI platforms currently remain in the research setting rather than being integrated into clinical practice.

The perceptions and commitment of doctors and their patients towards AI are critical factors for the further successful implementation of AI applications in healthcare ${ }^{[18-20]}$. Understanding the current knowledge and general attitude of surgeons toward AI applications in their surgical practice is essential and can contribute to the future uptake of AI in the surgical field. With this in mind, we aimed to investigate the existing knowledge, expectations, and concerns of AI among surgical residents and surgeons in the Netherlands.

\section{METHODS}

This survey was created using Google Forms and distributed for two weeks in March 2021 to the Dutch Association for Surgery (Nederlandse Vereniging voor Heelkunde) members by email. The questions were developed after review of the current literature and included after consultations with a core group of surgeons of the study ${ }^{[2,21-26]}$. Survey completion was voluntary and anonymous. The web-based survey consisted of 21 questions and was organized into five sections: four questions about demographics, four about AI knowledge, seven about the expectations of AI, four about the demands and concerns of AI, and two about the implementation of AI. It consisted of 13 multiple choice questions. In eight questions, we measured respondents' agreement with various statements on a five-point Likert scale ( $1=$ completely disagree, 2 = disagree, 3 = neutral, 4 = agree, 5 = completely agree). The study was performed in accordance 
with the ethical principles as stated in the Declaration of Helsinki.

\section{Statistical analyses}

The results are presented as proportions of the participants. For the Likert responses, mean composite scores were calculated based on the three related domains: knowledge, expectations, and concerns of AI. The Kruskal-Wallis test was used to detect differences according to the location of practice and license years. This test was used due to the non-normal distribution of the response. The Mann-Whitney U test was implemented to identify the specific group of differences. Statistical analysis was performed in IBM SPSS statistics 27 . A probability-value $(P$-value $)<0.05$ was considered significant.

\section{RESULTS}

A total of 313 respondents out of 1832 members of the Dutch Association for Surgery completed the survey (17\%), including 222 surgeons (71\%) and 91 surgical residents (29\%) [Table 1]. The majority of the surgeons [number $(n)=122,55 \%$ ] had more than 10 years of experience. Overall, 72\% worked in a district general hospital and $28 \%$ worked in an academic hospital. Of the surgeons, the most commonly noted subspecialty was gastrointestinal and oncological surgery followed by trauma surgery, vascular surgery, and pediatric surgery [Table 1]. The majority of the respondents indicated that they had not used AI in their hospital yet $(n=259,82.7 \%)$, and only a minority declared to actively follow developments of AI in professional and scientific journals $(n=84,27 \%)$ [Table 2]. Of the respondents, $40 \%$ indicated to be aware of the possibilities of AI ( $n=126$ agreed or wholly agreed) [Figure 1]. A substantial share of the respondents was interested in further education on artificial intelligence ( $n=215,69 \%$ agreed or completely agreed).

\section{Expectations and implementation of Al}

More than $50 \%$ of the respondents agreed to each of the following statements: "AI can be of value in the surgical field" (85\%); "I expect to improve my diagnostic ability with the help of AI" (61\%); and "The benefits of AI will outweigh the potential risks" (55\%) [Figure 1]. Fewer than half of the respondents expected that the help of AI would reduce complications (45\%), and $40 \%$ of the participants disagreed that AI could become superior to a surgeon's clinical experience. The respondents indicated the outpatient clinic $[n=112(35.8 \%)]$ and the operating room $[n=124(39.6 \%)]$ as the most valuable place for the implementation of AI [Table 2]. The greatest potential for AI applications in the operating room was expected in intraoperative image processing for surgical guidance $(57.5 \%)$, followed by automated performance metrics of surgeons (23.6\%). The great majority of the respondents highlighted the need for surgeons to participate in a multidisciplinary AI team at their own hospital (82.1\%) [Table 2]. Of the respondents, 50.8\% highlighted the need for the Dutch Association for Surgery in the Netherlands to fulfill an advisory and coordinating role, while $43.5 \%$ preferred a more proactive role in the development and implementation of AI.

\section{Risks and concerns of Al}

The great majority of the respondents (83.1\%) stated that the surgeon is responsible in the event a surgical complication arises while the surgeon uses an AI tool for surgical guidance. The top three most significant concerns about the use of AI were accountability issues (50.5\%), loss of autonomy (46.6\%), and risk of bias (43.5\%) [Figure 2]. A minority of the respondents (15.3\%) expressed their concerns on privacy issues, and only $11.5 \%$ of the respondents were concerned about the lack of scientific substantiation. Only $6.7 \%$ of the respondents agreed to be worried that AI will replace them at their job. Most survey respondents were not willing to undergo surgery on their gallbladder in the future when it is performed by a fully autonomous robot controlled by AI (78\%). 
Table 1. Demographic distribution of respondents

\begin{tabular}{lll}
\hline Demographic & $\boldsymbol{n}$ & (\%) \\
\hline Function & & $(71)$ \\
Surgeon & 222 & $(29)$ \\
Resident & 91 & $(28)$ \\
Surgeon's location of practice & & $(72)$ \\
Academic hospital & 63 & $(45)$ \\
District general hospital & 159 & $(36)$ \\
Surgeon's license years & & $(19)$ \\
$1-10$ years & 100 \\
$11-20$ years & 80 & $(57)$ \\
$>20$ years & 42 & $(20)$ \\
Surgeon's specialization & & $(19)$ \\
Gastrointestinal and oncological surgery & 127 \\
Traumatology & 45 & $(4)$ \\
Vascular surgery & 42 \\
Paediatric surgery & 8 & \\
\hline
\end{tabular}

\section{Subgroup analysis according to the characteristics of the surgeons}

Comparing the mean composite scores between the groups showed significant differences in the type of practice [Table 3]. Surgeons working in an academic hospital were significantly more likely to believe they have more knowledge of AI $(P=0.01)$ compared to the surgeons in district general hospitals. In response to the question considering the job replacement, surgeons in academic hospitals had significantly less concerns than surgeons in district general hospitals $(P=0.005)$. No significant differences were visible for the composite scores according to the years of surgical license.

\section{DISCUSSION}

Our survey provides preliminary insight into the perspectives of surgeons and surgical residents towards future artificial intelligent technologies in the surgical field. The findings demonstrate that the majority of the surgeons showed a positive and open attitude towards AI. Overall, the respondents largely agreed that AI could improve the quality of care in hospitals. The outpatient clinic and operating room were stated as the main areas of interest for the use of AI. To facilitate adequate adoption and implementation of AI, surgeons stated to be most willing to participate in multidisciplinary AI teams in hospitals. The expectations regarding the implementation of surgical AI applications were high, and surgeons were willing to undergo further learning.

The majority of the participants felt positive about the value ( $85 \%$ ) and diagnostic ability (61\%) of AI in the surgical field. In addition, it is encouraging that a greater share of the respondents believed that the benefits of AI applications will outweigh the risks. However, the respondents did see difficulties and concerns in utilizing AI in surgical care safely. The surgeons in this survey raised the greatest concern on liability issues. In general, medical malpractice law states that physicians provide a standard of care that can be expected of relevant members of the same specialty ${ }^{[27]}$. Since AI is not (yet) the legal standard of care, physicians are currently at risk of facing liability when injury results after following AI recommendation ${ }^{[28]}$. However, once $\mathrm{AI}$ is proven safe and of support to physicians, malpractice law might even require the use of $\mathrm{AI}^{[29]}$. In this survey, $83 \%$ of respondents felt that responsibility should be held with the surgeon. Defining the relevant standards of care will become a central issue in the further implementation of AI. Surgical societies can contribute by developing standardized datasets, stimulating research, and evaluating whether specific AI 
Table 2. Self-assessed knowledge, surgical expectations, and risks and concerns of Al

\begin{tabular}{|c|c|c|c|}
\hline \multirow{2}{*}{$\begin{array}{l}\text { Statement/question } \\
\text { Knowledge of Al }\end{array}$} & \multicolumn{3}{|l|}{ Responses $n(\%)$} \\
\hline & & & \\
\hline \multirow[t]{2}{*}{ I have made clinical use of $\mathrm{Al}$ in my hospital } & Yes & 54 & $(17.3)$ \\
\hline & No & 259 & $(82.7)$ \\
\hline \multirow[t]{2}{*}{ I actively follow the developments of $\mathrm{Al}$ in professional and scientific journals } & Yes & 84 & $(26.8)$ \\
\hline & No & 229 & $(73.2)$ \\
\hline \multicolumn{4}{|l|}{ Surgical expectations of Al } \\
\hline \multirow{4}{*}{$\begin{array}{l}\text { What department in your hospital could have the most significant potential value from } \\
\text { Al? }\end{array}$} & Outpatient clinic & 112 & $(35.8)$ \\
\hline & Operating room & 124 & $(39.6)$ \\
\hline & $\begin{array}{l}\text { Surgical ward/emergency } \\
\text { department }\end{array}$ & 44 & $(14.1)$ \\
\hline & Other & 33 & $(10.5)$ \\
\hline \multirow[t]{6}{*}{ What kind of application of $\mathrm{Al}$ in the operating room has the greatest potential? } & $\begin{array}{l}\text { Intraoperative image processing for } \\
\text { surgical }\end{array}$ & 180 & $(57.5)$ \\
\hline & Guidance & 74 & $(23.6)$ \\
\hline & $\begin{array}{l}\text { Automated performance metrics of } \\
\text { surgeons }\end{array}$ & 23 & $(7.3)$ \\
\hline & Black box & 18 & $(5.8)$ \\
\hline & $\begin{array}{l}\text { Surgical planning/efficiency of the } \\
\text { OR }\end{array}$ & 18 & $(5.8)$ \\
\hline & Other & & \\
\hline \multicolumn{4}{|l|}{ Implementation of AI } \\
\hline \multirow{4}{*}{$\begin{array}{l}\text { What role should the Dutch Association for surgery take in the development and } \\
\text { implementation of Al? }\end{array}$} & Advisory and coordinating task & 159 & $(50.8)$ \\
\hline & Active central role & 136 & $(43.5)$ \\
\hline & No role & 17 & $(5.4)$ \\
\hline & Other & 1 & $(0.3)$ \\
\hline \multicolumn{4}{|l|}{ Risks and concerns } \\
\hline \multirow{5}{*}{$\begin{array}{l}\text { Who should be held liable in the event of a complication, when the surgeon uses an Al } \\
\text { tool for surgical guidance? }\end{array}$} & Surgeon & 260 & $(83.1)$ \\
\hline & Hospital & 16 & $(5.1)$ \\
\hline & Company of the software/algorithm & 12 & $(3.8)$ \\
\hline & Patient, who signed consent & 5 & $(1.6)$ \\
\hline & Other & 20 & $(6.4)$ \\
\hline \multirow{2}{*}{$\begin{array}{l}\text { Are you willing to undergo surgery on your gallbladder performed by a future fully } \\
\text { autonomous robot controlled by Al? }\end{array}$} & Yes & 69 & $(22)$ \\
\hline & No & 244 & $(78)$ \\
\hline
\end{tabular}

Al: Artificial intelligence.

Table 3. Subgroup analysis according to the characteristics of the surgeons

\begin{tabular}{|c|c|c|c|c|c|c|c|}
\hline & \multicolumn{3}{|c|}{ License years } & \multirow[b]{2}{*}{$\boldsymbol{P}$} & \multicolumn{2}{|c|}{ Hospital } & \multirow[b]{2}{*}{$P$} \\
\hline & $\begin{array}{l}1-10 \text { years } \\
(n=100)\end{array}$ & $\begin{array}{c}11-20 \text { years } \\
(n=80)\end{array}$ & $\begin{array}{c}>20 \text { years } \\
(n=42)\end{array}$ & & $\begin{array}{l}\text { Academic hospital } \\
(n=63)\end{array}$ & $\begin{array}{l}\text { District general hospital } \\
(n=159)\end{array}$ & \\
\hline Knowledge $\left(^{\star}\right)$ & 3.5 & 3.6 & 3.4 & 0.191 & 3.7 & 3.5 & 0.01 \\
\hline Expectations $\left({ }^{\star \star}\right)$ & 3.6 & 3.7 & 3.5 & 0.27 & 3.6 & 3.6 & 0.896 \\
\hline Concerns $\left({ }^{\star \star \star}\right)$ & 1.7 & 1.6 & 1.6 & 0.839 & 1.4 & 1.7 & 0.005 \\
\hline
\end{tabular}

Mean composite score, based on Likert scale of 1-5: $1=$ completely disagree, $2=$ disagree, $3=$ neutral, $4=$ agree, $5=$ completely agree. $P$ values are based on Kruskal-Wallis test. ${ }^{\star} \mid$ am aware of the possibilities of Al, I am interested in further education about Al. ${ }^{* \star}$ The benefits of Al will outweigh the potential risks, I expect to reduce complications with help of Al, I expect to improve my diagnostic ability with help of $\mathrm{Al}$, $\mathrm{Al}$ can be of value in the surgical field, Al could become superior to a surgeon's clinical experience. ${ }^{\star \star \star} \mid$ am worried Al will replace my job. Al: Artificial intelligence. 


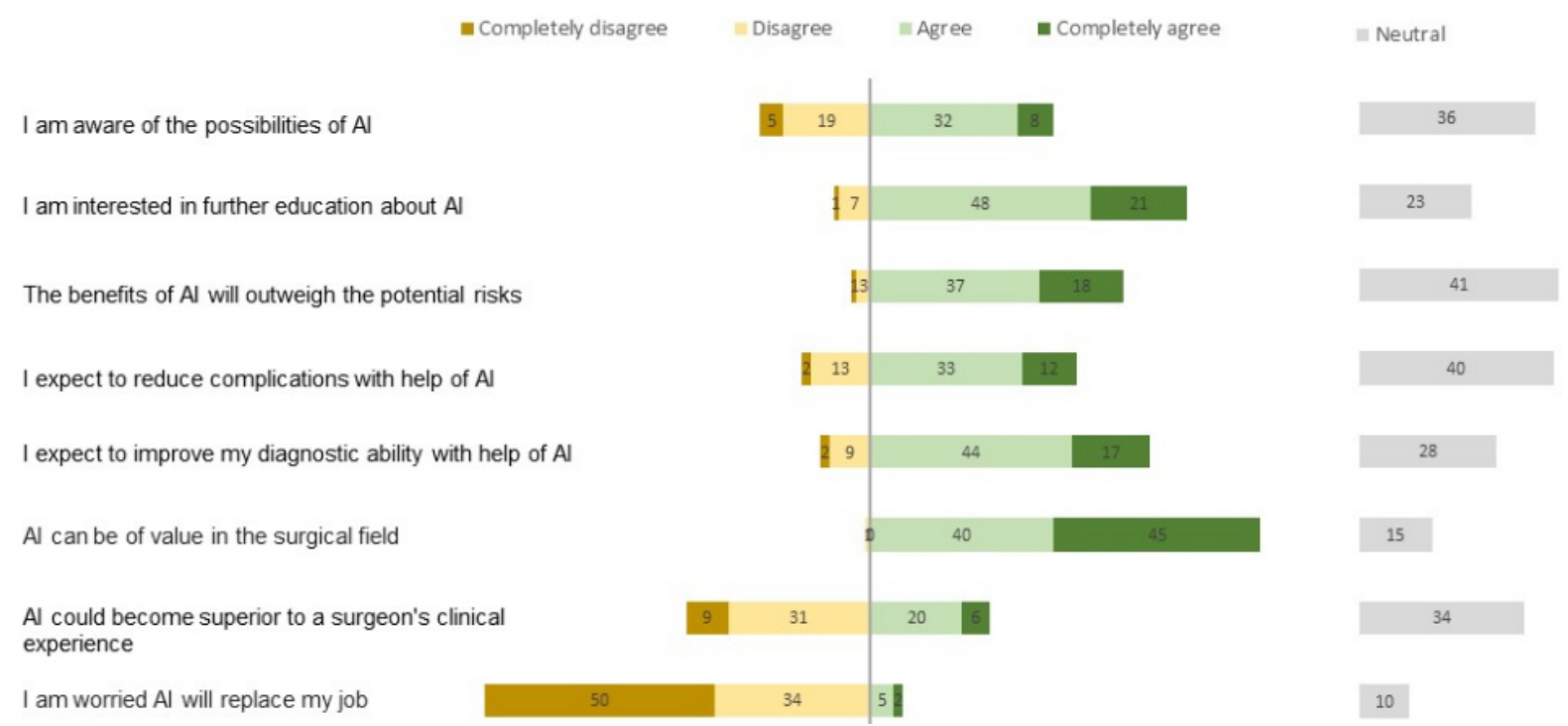

Figure 1. Respondents' expectations regarding Al. ${ }^{\star}$ The percentages may not add up to 100\% due to rounding. Al: Artificial intelligence.

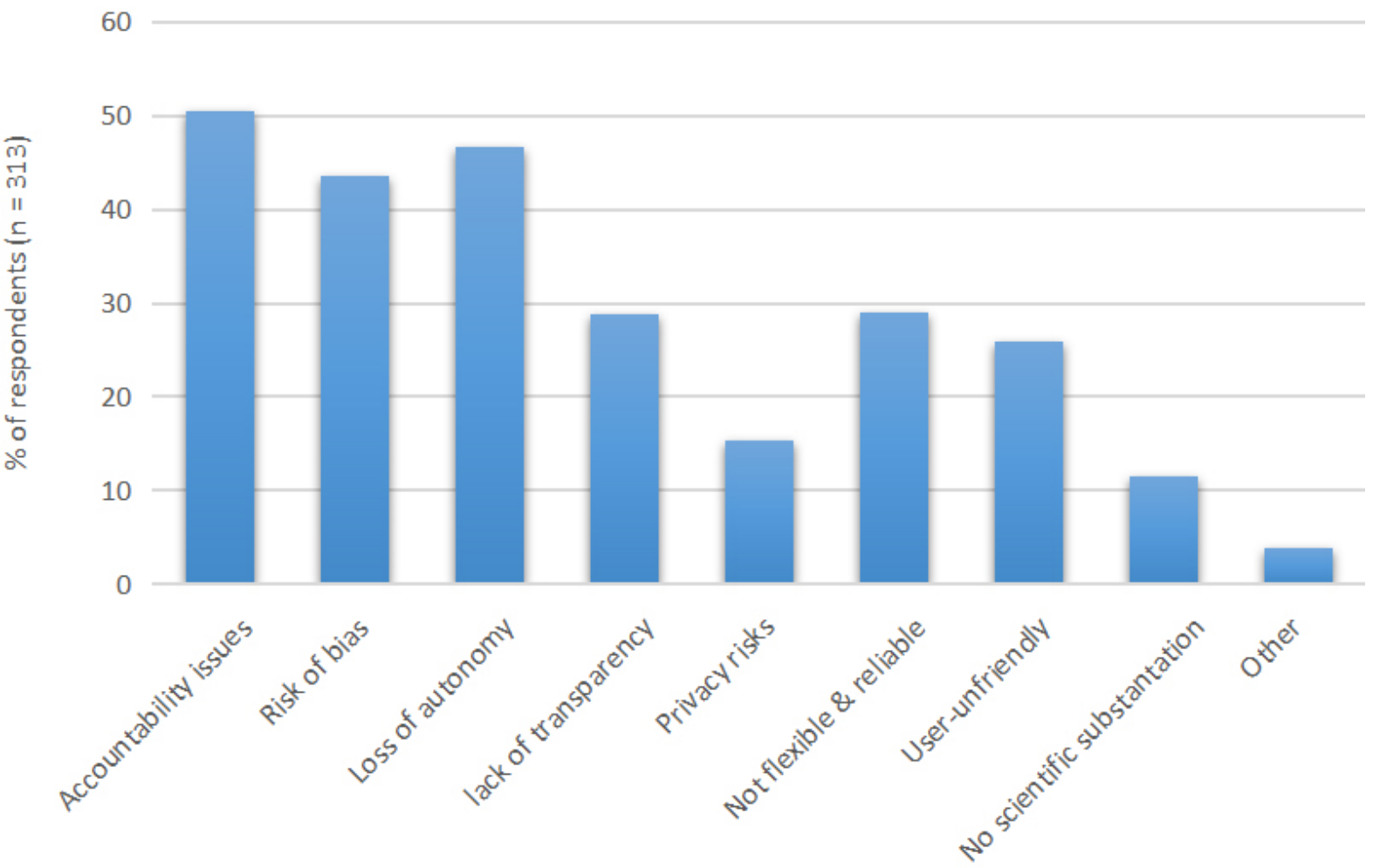

Figure 2. Showing the scoring responses for the perceived concerns of the use of artificial intelligence. The percentage does not equal $100 \%$, as it is a multiple-response question.

applications should be incorporated as the standard of care in practice guidelines ${ }^{[2]}$. In 2019, the Dutch Association for Surgery established an innovation working group with the mission to explore the current use and expectations of innovative developments in surgical practice. The working group covers, among other topics such as imaging technology, robotics, and biological imaging, the introduction and 
implementation of AI in surgery and stimulates partnerships with technical universities and companies. Early efforts of education can persuade surgeons to embrace clinically relevant AI development more easily. Involvement of surgical societies can resolve the threat of medical and legal implications and stimulate hospitals to deploy AI systems safely.

Interestingly, only $15 \%$ of the respondents noted privacy in their top three concerns. This finding is consistent with a previous study by Scheetz et al. ${ }^{[21]}$. In this survey of 632 trainees and fellows in ophthalmology, radiology, and dermatology, privacy did not belong to the top three ranked potential concerns. This could be explained by the fact that physicians have confidence in the current level of data protection and privacy at their hospitals. Another explanation could be the increasingly stringent data legislation. In 2018, the European Union took the lead in data legislation with the implementation of the General Data Protection Regulation (GDPR ${ }^{[3,3,3]}$. The GDPR mandates that adequate measures are taken to guarantee data security when personal data are collected, such as the provision of informed consent, improved transparency, and anonymization of data. Adequate legislation will be crucial for harmonizing AI in healthcare. The large volume of personal data that AI generates should be handled cautiously since a loss of trust from patients and physicians could result in a significant drawback for the implementation of $\mathrm{AI}^{[32]}$.

Surgeons in academic positions expressed a significantly higher knowledge of the possibilities of AI than their colleagues in district general hospitals. A clear explanation is not found, but the amount of education and research facilities in academic hospitals could play a role in this difference. Since the majority of AI applications are still in their research phase, most AI research programs will be enrolled more easily in academic medical centers. A previous survey on pathologists' perspectives of AI presented similar results, showing academic pathologists were significantly more optimistic about AI tool integration than their colleagues in community hospitals ${ }^{[33]}$.

The majority of the respondents considered the operating room as the desired setting for the deployment of AI tools. Regarding the potential for AI applications in the operating room, most respondents favored intraoperative image processing tools for surgical guidance. The remaining majority envisioned more potential in educational purposes through automated performance metrics of surgeons. Minimal invasive surgery appears to be most suitable for intraoperative analysis due to the great volume of (visual) data ${ }^{[26,34]}$. AI has been used on laparoscopic videos for phase recognition ${ }^{[35]}$, tool and gesture detection ${ }^{[36]}$, and identification of surgical anatomy ${ }^{[37,38]}$. However, the translation from research to the clinical implementation of AI in the operating room remains challenging ${ }^{[39]}$. Prospective participation of surgeons in model design and development will help researchers ensure that valuable clinical steps are taken.

Surgeons are less worried about job replacement compared to their colleagues in radiology. According to previous surveys in radiology and radiation oncology, $34 \%-42 \%$ of the physicians had a fear of (partial) replacement compared to $7 \%$ of the surgeons in this survey ${ }^{[2,25,40,41]}$. Surgeons' low concern on job replacement could be explained by the nature of their job, which requires fine complex manipulation during surgery and direct human interaction with patients. Occupations involving the complex perception of visual cues and delicate manipulation tasks in an unstructured work environment are considered unlikely to be substituted by algorithms in the near future ${ }^{[42]}$. The fact that surgeons are not susceptible to computerization is supported by a study of Frey et al ${ }^{[42]}$. In this study, a machine learning algorithm was applied to 702 jobs, and jobs were ranked according to their susceptibility to computerization. The algorithm used variables currently identified as bottlenecks in machine learning, including perception and manipulation, creative intelligence, and social intelligence tasks. While $47 \%$ of the job market was felt to be at risk for job losses, surgeons were least likely to be supplanted with an automation risk of $0.4 \%$. It is, therefore, unlikely that AI 
will fully replace surgeons in the foreseeable future. Perhaps the most important reason is that patients will always require human interaction and empathy ${ }^{[43]}$.

This survey has limitations. The first is the response rate of $17 \%$ of the Dutch Association for Surgery, of whom nearly $30 \%$ were surgical residents. Although this is comparable with previous online surveys, this could have resulted in selection bias ${ }^{[2,140]}$. Second, surgeons working in academic hospitals and surgeons with more than 20 years' experience were underrepresented. Some statistically significant differences were small, and one can question the clinical relevance. Thirdly, the respondents may represent disproportionately those surgeons with significant interest in artificial intelligence. Furthermore, respondents with limited knowledge of AI technology could have different and incorrect conceptualizations of AI. In our survey, only $17 \%$ of the respondents stated to make clinical use of AI. These findings are in line with previous surveys in other medical specialties, however this could have resulted in bias ${ }^{[2,44]}$. For example, surgeons could have confused AI with other innovative technologies, especially augmented reality. Finally, this questionnaire was designed for this survey, and its validity was not evaluated. The short, yet diverse range of questions in this survey does not obtain a fine-grained analysis of surgeons' opinions. Further research should address a more comprehensive account of the perceptions of surgeons, to obtain a richer understanding. Irrespective of these limitations, this survey provides interesting insights into surgeons' current views on AI in the surgical field. The majority of the surgeons have high expectations regarding future surgical AI applications. The respondents have highlighted various ethical issues and concerns but remain positive and have an open attitude towards AI. How AI will change the surgical landscape remains to be seen since AI is entering the domain of non-routine cognitive tasks. Technological developments are changing rapidly, and more and more AI applications are taken out into practice. Continued evaluation of physicians' perceptions is needed to ensure that data scientists will work on clinical valuable AI applications that meet the standards of their end users.

\section{DECLARATIONS}

\section{Acknowledgments}

The authors would like to thank Hillie Beumer for assisting with the distribution of the survey. Many thanks to all the surgical residents and surgeons who participated in this survey.

\section{Authors' contributions}

Developed the survey questions in consultation with all co-authors: Voskens FJ, Abbing JR

Contributed on data collection and data analysis and was the major contributor in writing the manuscript: Voskens FJ

Reviewed, edited and approved the final manuscript: Voskens FJ, Abbing JR, Ruys AT, Ruurda JP, Broeders IAMJ

All authors agreed to be personally accountable for the work.

\section{Availability of data and materials}

The data are available from the corresponding author on reasonable request.

\section{Financial support and sponsorship}

None.

\section{Conflicts of interest}

Ivo A. M. J. Broeders is a consultant surgeon for Johnson \& Johnson and Intuitive Surgical. Jelle P. Ruurda is a consultant for Intuitive Surgical. Frank J. Voskens, Anthony T. Ruys and Julian R. Abbing have no conflicts of interest or financial ties to disclose. 


\section{Ethical approval and consent to participate}

The study was performed in accordance with the ethical principles as stated in the Declaration of Helsinki. Ethical approval was not required, as the Dutch Medical Research on Humans Act (WMO) did not apply to this study. The present study did not include patients, participation was voluntary and did not bring along any risk. Returning the digital questionnaire was interpreted as informed consent to participate in the study.

\section{Consent for publication}

Not applicable.

\section{Copyright}

(c) The Author(s) 2022.

\section{REFERENCES}

1. Bellman R. An introduction to artificial intelligence: can computers think? Available from: http://www.researchgate.net/publication/239053505_An_Introduction_to_Artificial_Intelligence--Can_Computers_Think [Last accessed on 30 Dec 2021].

2. Hashimoto DA, Rosman G, Rus D, Meireles OR. Artificial intelligence in surgery: promises and perils. Ann Surg 2018;268:70-6. DOI PubMed PMC

3. LeCun Y, Bengio Y, Hinton G. Deep learning. Nature 2015;521:436-44. DOI PubMed

4. Gupta A, Anpalagan A, Guan L, Khwaja AS. Deep learning for object detection and scene perception in self-driving cars: survey, challenges, and open issues. Array 2021;10:100057. DOI

5. Ayala Solares JR, Diletta Raimondi FE, Zhu Y, et al. Deep learning for electronic health records: a comparative review of multiple deep neural architectures. J Biomed Inform 2020;101:103337. DOI PubMed

6. Wang D, Khosla A, Gargeya R, Irshad H, Beck AH. Deep learning for identifying metastatic breast cancer. arXiv preprint arXiv 2016:1606.05718. DOI

7. Yu KH, Beam AL, Kohane IS. Artificial intelligence in healthcare. Nat Biomed Eng 2018;2:719-31. DOI PubMed

8. Mckinney SM, Sieniek M, Godbole V, et al. International evaluation of an AI system for breast cancer screening. Nature 2020;577:8994. DOI PubMed

9. Lakhani P, Sundaram B. Deep learning at chest radiography: automated classification of pulmonary tuberculosis by using convolutional neural networks. Radiology 2017;284:574-82. DOI PubMed

10. Liu Y, Kohlberger T, Norouzi M, et al. Artificial intelligence-based breast cancer nodal metastasis detection: insights into the black box for pathologists. Arch Pathol Lab Med 2019;143:859-68. DOI PubMed

11. Coudray N, Ocampo PS, Sakellaropoulos T, et al. Classification and mutation prediction from non-small cell lung cancer histopathology images using deep learning. Nat Med 2018;24:1559-67. DOI PubMed

12. Ting DSW, Pasquale LR, Peng L, et al. Artificial intelligence and deep learning in ophthalmology. Br J Ophthalmol 2019;103:167-75. DOI PubMed PMC

13. Gulshan V, Peng L, Coram M, et al. Development and validation of a deep learning algorithm for detection of diabetic retinopathy in retinal fundus photographs. JAMA 2016;316:2402-10. DOI PubMed

14. Haenssle HA, Fink C, Schneiderbauer R, et al; Reader study level-I and level-II Groups. Man against machine: diagnostic performance of a deep learning convolutional neural network for dermoscopic melanoma recognition in comparison to 58 dermatologists. Ann Oncol 2018;29:1836-42. DOI PubMed

15. Esteva A, Kuprel B, Novoa RA, et al. Dermatologist-level classification of skin cancer with deep neural networks. Nature 2017;542:115-8. DOI PubMed PMC

16. Mirnezami R, Ahmed A. Surgery 3.0, artificial intelligence and the next-generation surgeon. Br J Surg 2018;105:463-5. DOI PubMed

17. Zhou XY, Guo Y, Shen M, Yang GZ. Application of artificial intelligence in surgery. Front Med 2020;14:417-30. DOI PubMed

18. Esmaeilzadeh P. Use of AI-based tools for healthcare purposes: a survey study from consumers' perspectives. BMC Med Inform Decis Mak 2020;20:170. DOI PubMed PMC

19. Davenport T, Kalakota R. The potential for artificial intelligence in healthcare. Future Healthc J 2019;6:94-8. DOI PubMed PMC

20. Drysdale E, Dolatabadi E, Chivers C, et al. Implementing AI in healthcare. Available from: http://www.erikdrysdale.com/figures/implementing-ai-in-healthcare.pdf [Last accessed on 30 Dec 2021].

21. Scheetz J, Rothschild P, McGuinness M, et al. A survey of clinicians on the use of artificial intelligence in ophthalmology, dermatology, radiology and radiation oncology. Sci Rep 2021;11:5193. DOI PubMed PMC

22. van Hoek J, Huber A, Leichtle A, et al. A survey on the future of radiology among radiologists, medical students and surgeons: students and surgeons tend to be more skeptical about artificial intelligence and radiologists may fear that other disciplines take over. Eur J Radiol 2019;121:108742. DOI PubMed

23. European Society of Radiology (ESR). Impact of artificial intelligence on radiology: a EuroAIM survey among members of the European Society of Radiology. Insights Imaging 2019;10:105. DOI

24. Shinners L, Aggar C, Grace S, Smith S. Exploring healthcare professionals' perceptions of artificial intelligence: validating a 
questionnaire using the e-Delphi method. Digit Health 2021;7:20552076211003433. DOI PubMed PMC

25. Huisman M, Ranschaert E, Parker W, et al. An international survey on AI in radiology in 1,041 radiologists and radiology residents part 1: fear of replacement, knowledge, and attitude. Eur Radiol 2021;31:7058-66. DOI PubMed PMC

26. Birkhoff DC, van Dalen ASHM, Schijven MP. A review on the current applications of artificial intelligence in the operating room. Surg Innov 2021;28:611-9. DOI PubMed PMC

27. Epstein NE. Legal and evidenced-based definitions of standard of care: Implications for code of ethics of professional medical societies. Surg Neurol Int 2018;9:255. DOI PubMed PMC

28. Price WN 2nd, Gerke S, Cohen IG. Potential liability for physicians using artificial intelligence. JAMA 2019;322:1765-6. DOI PubMed

29. Froomkin AM, Kerr I, Pineau J. When AIs outperform doctors: confronting the challenges of a tort-induced over-reliance on machine learning. Ariz L Rev 2019;61:33. DOI

30. Forcier MB, Gallois H, Mullan S, Joly Y. Integrating artificial intelligence into health care through data access: can the GDPR act as a beacon for policymakers? J Law Biosci 2019;6:317-35. DOI PubMed PMC

31. Pesapane F, Volonté C, Codari M, Sardanelli F. Artificial intelligence as a medical device in radiology: ethical and regulatory issues in Europe and the United States. Insights Imaging 2018;9:745-53. DOI PubMed PMC

32. Asan O, Bayrak AE, Choudhury A. Artificial intelligence and human trust in healthcare: focus on clinicians. J Med Internet Res 2020;22:e15154. DOI PubMed PMC

33. Sarwar S, Dent A, Faust K, et al. Physician perspectives on integration of artificial intelligence into diagnostic pathology. NPJ Digit Med 2019;2:28. DOI PubMed PMC

34. Kawka M, Gall TM, Fang C, Liu R, Jiao LR. Intraoperative video analysis and machine learning models will change the future of surgical training. Intelligent Surgery 2021. DOI

35. Padoy N. Machine and deep learning for workflow recognition during surgery. Minim Invasive Ther Allied Technol 2019;28:82-90. DOI PubMed

36. Luongo F, Hakim R, Nguyen JH, Anandkumar A, Hung AJ. Deep learning-based computer vision to recognize and classify suturing gestures in robot-assisted surgery. Surgery 2021;169:1240-4. DOI PubMed PMC

37. Tokuyasu T, Iwashita Y, Matsunobu Y, et al. Development of an artificial intelligence system using deep learning to indicate anatomical landmarks during laparoscopic cholecystectomy. Surg Endosc 2021;35:1651-8. DOI PubMed PMC

38. Madani A, Namazi B, Altieri MS, et al. Artificial intelligence for intraoperative guidance: using semantic segmentation to identify surgical anatomy during laparoscopic cholecystectomy. Ann Surg 2020. DOI PubMed PMC

39. Kelly CJ, Karthikesalingam A, Suleyman M, Corrado G, King D. Key challenges for delivering clinical impact with artificial intelligence. BMC Med 2019;17:195. DOI PubMed PMC

40. Oh S, Kim JH, Choi SW, Lee HJ, Hong J, Kwon SH. Physician confidence in artificial intelligence: an online mobile survey. J Med Internet Res 2019;21:e12422. DOI PubMed PMC

41. Wong K, Gallant F, Szumacher E. Perceptions of Canadian radiation oncologists, radiation physicists, radiation therapists and radiation trainees about the impact of artificial intelligence in radiation oncology - national survey. J Med Imaging Radiat Sci 2021;52:44-8. DOI PubMed

42. Frey CB, Osborne MA. The future of employment: how susceptible are jobs to computerisation? Technol Forecast Soc Change 2017;114:254-80. DOI

43. Krittanawong C. The rise of artificial intelligence and the uncertain future for physicians. Eur J Intern Med 2018;48:e13-4. DOI PubMed

44. Staartjes VE, Stumpo V, Kernbach JM, et al. Machine learning in neurosurgery: a global survey. Acta Neurochir (Wien) 2020;162:3081-91. DOI PubMed PMC 